\title{
Investigation of possible tolerance mutations in a VapBC Toxin-Antitoxin system through structure determination
}

\author{
Maria Raadkjær Nielsen and Ditlev E. Brodersen \\ Department of Molecular Biology and Genetics, Aarhus University, Gustav Wieds Vej 10, DK-8000, Aarhus C, Denmark \\ mrn@mbg.au.dk
}

Bacterial chromosomes contain large numbers of toxin-antitoxin (TA) systems, consisting of a gene encoding a toxic protein and a gene encoding an antitoxin which can be an RNA or a protein [1,2]. In the largest known group of type II TA systems, vapBC, the toxin VapC is an endoribonuclease belonging to the PIN (PilT N-terminal) domain family, which is very conserved structurally and Keywords: Toxin-antitoxin systems, tolerance, crystallographyKeywords: Toxin-antitoxin systems, tolerance, crystallographyKeywords: Toxin-antitoxin systems, tolerance, crystallographyfound in all domains of life. VapC is a known RNase and targets for VapCs include various tRNAs and rRNAs. The VapB antitoxin inhibits the toxin with its $\mathrm{C}$ terminus domain and contains a DNA binding domain in the $\mathrm{N}$ terminus [1].

TA systems have been implicated in bacterial tolerance to antibiotics, which can eventually lead to antibiotic resistance and it is considered a major health challenge by the WHO [3]. Previously, it was experimentally observed when a bacterial strain developed antibiotic tolerance in the presence of ampicillin during intermittent exposure, the development of tolerance increase the risk of the strain also developing antibiotic resistance [4]. In strains of Escherichia coli KLY where tolerance was observed, it was shown that mutations in a gene encoding VapB were present [4].

However, it is not known how or if TA systems are involved in creating tolerance and potentially affect antibiotic resistance. Here, we determine the structure of VapBC from E. coli KLY to $2.8 \AA$ using x-ray crystallography. The structure is overall very similar to the previously determined structure of a VapBC complex from Shigella flexneri 2a, where the VapBC is encoded on the pMYSH6000 plasmid. This VapBC was shown to form a hetero-octameric complex containing four VapB and four VapC proteins [5]. The VapBC from E. coli KLY differs from the VapBC in S. flexneri 2a in six positions. The two VapBs have two amino acids difference and the VapCs have four amino acid differences among them.

In the near future, structures representing several VapBC complexes with naturally occurring tolerance mutations will be determined. We envisage that the information gained will allow us to hypothesize the possible functional implications of the mutations and the possible effect this could have on the bacterium and whether this could explain an antibiotic tolerant phenotype.

[1] Bendtsen, K.L. and D.E. Brodersen, Higher-Order Structure in Bacterial VapBC Toxin-Antitoxin Complexes. Subcell Biochem, 2017. 83: p. 381412.

[1] Harms, A., et al., Toxins, Targets, and Triggers: An Overview of Toxin-Antitoxin Biology. Molecular Cell, 2018. 70(5): p. 768-784.

[1] Antimicrobial resistance. Available from: https://www.who.int/health-topics/antimicrobial-resistance.

[1] Levin-Reisman, I., et al., Antibiotic tolerance facilitates the evolution of resistance. Science, 2017. 355(6327): p. 826-830.

[1] Dienemann, C., et al., Crystal structure of the VapBC toxin-antitoxin complex from Shigella flexneri reveals a hetero-octameric DNA-binding assembly. J Mol Biol, 2011. 414(5): p. 713-22.

Keywords: Toxin-antitoxin systems, tolerance, crystallography 